\title{
Polymers in Curved Boxes
}

\author{
K. Yaman*, P. Pincus \\ Department of Physics, University of California \\ Santa Barbara, CA 93106-9530 \\ F. Solis, T.A. Witten \\ James Franck Institute, University of Chicago \\ 5640 Ellis Avenue, Chicago, IL 60637
}

(November 10, 2018)

\begin{abstract}
We apply results derived in other contexts for the spectrum of the Laplace operator in curved geometries to the study of an ideal polymer chain confined to a spherical annulus in arbitrary space dimension $\mathrm{D}$ and conclude that the free energy compared to its value for an uncurved box of the same thickness and volume, is lower when $D<3$, stays the same when $D=3$, and is higher when $D>3$. Thus confining an ideal polymer chain to a cylindrical shell, lowers the effective bending elasticity of the walls, and might induce spontaneous symmetry breaking, i.e. bending. (Actually, the above mentioned results show that any shell in $D=3$ induces this effect, except for a spherical shell). We compute the contribution of this effect to the bending rigidities in the Helfrich free energy expression.
\end{abstract}




\section{INTRODUCTION}

The quantum mechanics of particles confined to surfaces or surface layers embedded in a higher dimensional space, e.g. curves, tubular structures around curves, surfaces or layers between two surfaces in three dimensions, has attracted attention from many authors in the past three decades. Recently there has been considerable progress in rigorously, and non-perturbatively analyzing the changes to the spectrum of the Laplacian in curved geometries in two dimensions - these have been motivated by applications in mesoscopic semi-conductor physics, [四], [2]. There are also studies of the contribution of torsion to the bound state energies, [3], [4]. Until now the results for higher dimensional confinement are restricted to the perturbative regime, [5], [6], [7], [8], [9], [3], [10].

Below we summarize the main results of these studies, as they relate to the application of interest here. The next section reviews the relation between quantum mechanics and polymer physics in some detail. We compute the free energy in the geometries of interest in the third section. Most of the details of these calculations are left to an appendix. The final section computes the effective bending rigidities of a dilute polymer solution confined in a box with elastic walls, and includes our concluding remarks.

The correct quantization procedure for quantum particles confined to manifolds embed-

ded in higher dimensional spaces turns out to be non-trivial, [5]. Studies, [6] - [12], which are perturbative in the curvature of the manifold, conclude that confinement to a curved manifold results in an effective potential, which depends both on the manifold to which the particle is confined and the particular way this manifold is embedded in the higher dimensional space, i.e. both on the intrinsic and extrinsic curvatures of the manifold. This dependence on the extrinsic curvature means that one cannot arrive at the correct Schrödinger's equation by just transforming the Laplacian to the coordinates of the confining manifold; this can be understood in the light of the uncertainty principle - the particle will know that there is an "outside", no matter how strongly it is confined.

Confinement to a curve in three dimensions generates a potential, which is attractive to 
the most highly curved regions, in the one dimensional Schrödinger's equation; this potential is proportional to the square of the local curvature of the curve, [11]. If one considers a 'thintube' that is wound around a curve and has constant cross-section, (see Fig.1), to lowest order in the curvature one finds the same result: the energy change is proportional to the square of the curvature of the curve that the tube follows. The proportionality constant is independent of the shape of the cross-section, and also of the exact details of the confining potential (hardwalls etc.), [7], [3], and [13] - this can be easily understood by dimensional analysis, scaling arguments and using the result of [1]. Confinement between two equidistant surfaces in general dimension results in a potential $U$, which (in the appropriate units, i.e. neglecting overall positive constants) is given to second order in the principal radii of curvatures $R_{i}$, $(i=1,2,3, . .,(D-1))$, by [6]:

$$
U=-\frac{1}{4}\left(2 \sum_{i} \frac{1}{R_{i}^{2}}-\left(\sum_{i} \frac{1}{R_{i}}\right)^{2}\right) .
$$

For a spherical shell embedded in $D$ dimensions one sets $R_{i}=a$, for all $i$, where $a$ is the radius of the shell, and, as first written down in [10], Eq.(1) reduces to $U=\frac{(D-1)(D-3)}{a^{2}}$. This is negative for $1<D<3$.

Of course, all of the above results apply to any physical system that contains the Laplacian in its equations of motion.

\section{CONNECTION TO POLYMER PHYSICS}

The particle-in-a-box problem of quantum mechanics is closely related to confined ideal polymers. A flexible polymer in solution is a random walk. In the case without excluded volume interactions this will be denoted as an 'ideal chain'; i.e. ideal chains are allowed to self-intersect without raising their energy. An ideal polymer chain configuration may be mapped onto the motion of a quantum particle, where the probability distribution function is the analogue of the wave function, and the degree of polymerization $(\mathrm{N})$ plays the role of

imaginary time [15]. In this spirit we reduce the 'ideal-chain-in-a-box' problem to a quantum 'particle-in-a-box' problem. 
We consider a random walk in space, and work in the continuum description. Let $G\left(\vec{r}, \vec{r}^{\prime}, N\right)$ be the probability for a walk of $N$ steps to have its ends at $\vec{r}$ and $\vec{r}^{\prime}$. The function $G$ is defined to be 0 for $N<0$, and it satisfies [15] the differential equation:

$$
\left(\frac{\partial}{\partial N}-\frac{l^{2}}{6} \nabla_{\vec{r}}^{2}\right) G\left(\vec{r}, \vec{r}^{\prime}, N\right)=\delta\left(\vec{r}-\vec{r}^{\prime}\right) \delta(N) .
$$

Here $l$ is the monomer length. Eq. (2) describes diffusion once the boundary conditions are specified. We may also think about it as Schrödinger's equation, where $N$ plays the role of imaginary time.

The partition function is just a sum over all configurations:

$$
Z=(1 / V) \int d \vec{r} d \vec{r}^{\prime} G\left(\vec{r}, \vec{r}^{\prime}, N\right)
$$

The factor of $1 / V$ keeps the volume fixed when the free energy is computed.

Here we comment on the exact problem that we are considering, and our choice of boundary conditions, when we compare the flat and curved boxes. Our main aim is to find out the effects of polymer confinement on the bending elasticities of the confining structure. Hence we would like to isolate the change in the free energy due to curvature only. Therefore we pick periodic boundary conditions in the transverse directions of the flat box. Also to the same end we conserve the volume of the box during bending; this, of course, will fix the radius of the curved box once the amount of material (the surface area of the flat box) is fixed. Assuming that the free energy per unit area for this system can be expanded in the curvature when this radius is large compared to the thickness of the box, one can compute the coefficients in this expansion to second order in the curvature. There are in general two bending elasticities to compute. These can be uniquely determined from the two equations one attains by considering the free energy of the sphere and the cylinder. We have in mind membranes which generally do not have free edges but end at the walls of a container, or are closed vesicles. Then, e.g. for a container with repulsive walls, one has to take into account when computing the energy balance the fact that the eigenfunctions for the flat box vanish at the transverse edges. Also, the volume for the real system may not be conserved 
- this would bring translational entropy pieces into the energy balance in addition to the contributions we calculate in the present work.

To compute the partition function we use the method of eigenfunctions and levels of the Schrödinger's equation. This is an 'effective Hamiltonian' method where one integrates out certain configurations of the polymer chain and labels the coarse-grained modes by discrete 'levels'. Thus we write $Z=\sum_{n} e^{-\frac{\epsilon_{n}}{k_{B} T}}$, where the sum is over the levels and $\epsilon_{n}$ 's are the eigenvalues of the effective Hamiltonian that describes the system in terms of these levels only. For the remainder of this paper, in order to simplify the notation, we measure all energies in the units of $N k_{B} T$, and all lengths in the units of $(l / \sqrt{6})$, (except in the final results). It becomes clear what these levels are once one employs the eigenfunction expansion [16] for the propagator of the chain: $G\left(\vec{r}, \vec{r}^{\prime}, N\right)=\sum_{n} \Psi_{n}^{*}(\vec{r}) \Psi_{n}\left(\vec{r}^{\prime}\right) e^{-N E_{n}} \Theta(N)$. Here $\Theta(N)$ is the step function assuring that $G=0$ for $N<0$, and the $\Psi_{n}(\vec{r})$ 's are normalized solutions to $\nabla^{2} \Psi(\vec{r})=-E \Psi(\vec{r})$. It is easy to verify that this expansion indeed solves Eq.(2)), (recall: $\left.\frac{\partial}{\partial N} \Theta(N)=\delta(N)\right)$. The boundary conditions are $\Psi(\vec{r})=0$ at the walls which are at $|\vec{r}|=a$ and $|\vec{r}|=a+d$. This is a slab of thickness $d$ in one dimension, and a spherical shell of radius $a$ and thickness $d$ in any dimension $D$.

Using this eigenfunction expansion in the partition function expression, Eq.(3)), we find:

$$
Z=(1 / V) \sum_{n} e^{-N E_{n}} \int d \vec{r} \Psi_{n}^{*}(\vec{r}) \int d \overrightarrow{r^{\prime}} \Psi_{n}\left(\overrightarrow{r^{\prime}}\right)=(1 / V) \sum_{n} e^{-N E_{n}}\left|\int d \vec{r} \Psi_{n}(\vec{r})\right|^{2} .
$$

We see that in the geometry of interest all states except the spherically symmetric ones disappear from the sum in Eq. (四), even though they do contribute to the propagator. (In $D=1$, the sinusoidal solutions with an even number of peaks disappear). Hence:

$$
Z=(1 / V) \sum_{n=1}^{\infty} e^{-N E_{n}(a, d)}\left|\int d r r^{(D-1)} R_{n}(r)\right|^{2}
$$

where $n$ labels the distinct s-wave levels, $R_{n}(r)$ is the normalized radial wavefunction, and we explicitly indicate the dependence of the energies on $a$ and $d$. Defining:

$$
B_{n}(a, d) \equiv \frac{\left|\int d r r^{(D-1)} R_{n}(r)\right|^{2}}{\frac{S_{D-1}}{D}\left((a+d)^{D}-a^{D}\right)}
$$


where $S_{D-1}$ is the surface area of the unit sphere in $D$ dimensions, we can write for the free energy:

$$
F=-k_{B} T M \log \left(\sum_{n=1}^{\infty} e^{-\left(E_{n}(a, d) N l^{2} / 6\right)} B_{n}(a, d)\right)
$$

where $\mathrm{M}$ is the number of chains (each containing $\mathrm{N}$ links) in the box.

We now proceed with the analysis of the above expression.

\section{FREE ENERGY FOR POLYMERS IN BOXES}

We consider a spherical annulus of large radius and small thickness in arbitrary dimension D. The walls are taken to be repulsive for the polymer. Physical interest lies in $D=2$ (Fig. 2), which is a cylindrical shell in three dimensions], and $D=3$ (Fig. 3), a spherical shell in three dimensions.

The Laplacian in D-dimensions is $\nabla^{2}=\frac{d^{2}}{d r^{2}}+\frac{(D-1)}{r} \frac{d}{d r}-\frac{\vec{L}^{2}}{r^{2}}$. Here $\vec{L}$ is the angular momentum operator in $D$ dimensions. After standard manipulations we find the following quantization condition in terms of dimensionless variables:

$$
J_{\xi}\left[(\tilde{E})^{\frac{1}{2}} \frac{1}{\epsilon}\right] N_{\xi}\left[(\tilde{E})^{\frac{1}{2}}\left(1+\frac{1}{\epsilon}\right)\right]-J_{\xi}\left[(\tilde{E})^{\frac{1}{2}}\left(1+\frac{1}{\epsilon}\right)\right] N_{\xi}\left[(\tilde{E})^{\frac{1}{2}} \frac{1}{\epsilon}\right]=0,
$$

where $\tilde{E} \equiv E d^{2}, \xi^{2} \equiv \nu^{2}+\left(\frac{D-2}{2}\right)^{2}, \epsilon \equiv\left(\frac{d}{a}\right)$, and $\nu^{2}$ is an eigenvalue of the $S O(D)$ symmetric Casimir operator, and the functions $J$ and $N$ are the standard Bessel functions. As explained earlier, spherical symmetry of the geometries considered causes all but the $\nu=0$ states to drop out of the partition function. Defining $\mu \equiv 4 \xi^{2}$, (for $\nu=0$ ), $\mu=(D-2)^{2}$, and labelling these s-wave states by $n=1,2, \ldots$, one can now proceed with a perturbative analysis by expanding Eq.(8) in powers of $\epsilon=\frac{d}{a}$, which results in:

\footnotetext{
${ }^{1}$ The length along the axis of the cylinder is taken to be large compared to the other dimensions, and therefore does not play an essential role here.

${ }^{2} \mathrm{SO}(\mathrm{D})$ is the rotation group in $D$ dimensions.
} 


$$
d^{2} E_{n}(a, d)=(n \pi)^{2}+\frac{(D-1)(D-3)}{4}\left[\left(\frac{d}{a}\right)^{2}-\left(\frac{d}{a}\right)^{3}+\left(\frac{d}{a}\right)^{4}\left(1-\frac{3}{8 n^{2} \pi^{2}}\right)+\cdots\right] .
$$

This is the same perturbative result cited above. We see that the curvature lowers the spherically symmetric energy spectrum for $1<D<3$, does not affect it when $D=1$ (trivial), or $D=3$, and increases it everywhere else. Notice that the perturbation series stops for $D=1$, and $D=3$. Also notice that the perturbative effect is the same for all modes up to fourth order, this simplifies treating the full free energy of the polymer, Eq.([7). This treatment an expression for $B_{n}(a, d)$, the computation of which is difficult in general, but we were able to find an exact expression in $D=3$, and a perturbative one in $D=2$. We now summarize our results. Details are left to the appendix.

\section{A. Three Dimensions}

Defining $Z_{0}$ as the partition function of the flat box, and $k \equiv\left(N l^{2} \pi^{2} / 6 d^{2}\right)$ we find the exact result for the partition function:

$$
Z / Z_{0}=1+g(\epsilon)(1-3 f(k))
$$

where the functions $f$ and $g$ are defined in the appendix. One can expand the above expressions in two limits: when the typical polymer dimension, $R_{g} \equiv \sqrt{N} l$, is smaller or larger than the box width, $d$. The latter is the so-called ground state dominance regime (G.S.D.) - here $k>1$; the former we will denote by "the colloid limit" (C.L.), since in this limit the coils look like small balls - here $k<1$. In G.S.D., the corrections due to $B_{n}$ are negligible, and we find that the free energy does not have any corrections due to curvature. In C.L. we find:

$\Delta F=k_{B} T M \frac{1}{12}\left(\frac{d}{a}\right)^{2}\left(\frac{8}{\pi^{1 / 2}} \sqrt{\frac{N l^{2}}{6 d^{2}}}-\frac{4(3 \pi-8)}{\pi} \frac{N l^{2}}{6 d^{2}}-\frac{16(3 \pi-8)}{\pi^{3 / 2}}\left(\frac{N l^{2}}{6 d^{2}}\right)^{3 / 2}+\cdots\right)$

\section{B. Two Dimensions}

In this case we find (to lowest order in $\epsilon$ ): 


$$
Z / Z_{0}=e^{+\frac{k \epsilon^{2}}{4 \pi^{2}}}\left(1-\frac{\epsilon^{2}}{16}\left(1-f(k)-\frac{8}{\pi^{2}} h(k)\right)+\cdots\right)
$$

where $f$ is the same function as for three dimensions, and $h$ is defined in the appendix. This, in G.S.D. leads to:

$\Delta F=-k_{B} T M N \frac{1}{24} \frac{l^{2}}{a^{2}}$.

In C.L. there are no terms of order $\sqrt{k}$, and two terms of order $k$ which cancel each other exactly, and the expansion starts at third order, (this is similar to what happens for small hard spheres, i.e. colloids, - there one finds that there is no shift in the free energy in two dimensions, and a positive shift in three dimensions, [17]):

$\Delta F=-k_{B} T M \frac{1}{18 \sqrt{6 \pi}}\left(\frac{d}{a}\right)^{2}\left(\frac{N l^{2}}{d^{2}}\right)^{3 / 2}+\cdots$

In the strong confinement (G.S.D.) regime it does not matter to which chain the monomers belong - the shift in the energy is proportional to the total number of monomers, $M N$; in the C.L. regime we have an ideal gas of $M$ objects.

We comment on some issues before computing effective bending rigidities using the above results: In the non-perturbative regime we solved the eigenvalue equation, Eq. (8), numerically for the ground states in $D=2,4$, and 5 , for $2<\frac{a}{d}<50$. The large $\frac{a}{d}$ behavior of the solutions, (see the curves plotted in Fig. 4), are exactlyf as predicted by Eq. (9). Notice that they are all converging to the flat case value of $\pi^{2}$ for very small curvature. As the curvature increases the behavior changes its functional form, and becomes much sharper than just a quadratic deviation. One can follow these curves farther towards infinite curvature, and see that they tend to a constant value that depends on the dimensionality. This pattern is also valid for $n \neq 1, \nu=0$ (s-wave), states.

For surfaces in three dimensions Eq.(1) reduces to: $U=-\frac{1}{4}\left(\frac{1}{R_{1}}-\frac{1}{R_{2}}\right)^{2}$. The effect of this potential, even after integration over the entire surface, is always attractive except for in two cases where it has no effect at all, the flat surface, and the sphere. Notice also that in $D=2$ one always gets an attractive potential if the confining curves are not perfectly

\footnotetext{
${ }^{3}$ This is not obvious from the plot given here.
} 
flat. Thus in two dimensions, -(physically we think about generalized cylinders living in three dimensions), the free energy is reduced by any curvature, regardless of the shape of the boundaries. Although we are not considering large curvatures here, it is worthwhile to mention that the literature cited in the introduction proves that in one and two dimensional structures, (tubes and strips), the ground state energy of the Laplace operator decreases due to curvature, and there is a bound state localized in the regions of curvature. This is a non-perturbative statement, and it makes very weak assumptions about the detailed geometry. This is important because it implies that in these systems, (at least in G.S.D.), the polymer would localize in regions where there is curvature. One explicit example of this is the numerical solution of Schrödinger's equation in oval shaped rings [14.

\section{EFFECT ON $\kappa$ AND $\kappa$}

We conclude by calculating the contribution of this effect to the effective bending curvature elasticity of an ideal polymer confined to a fluid bilayer membrane. Let $f_{c}$ be the curvature free energy per area. Up to quadratic order in the principal radii $\frac{1}{R_{1}}$ and $\frac{1}{R_{2}}, f_{c}$ can be written in terms of the mean and Gaussian curvatures of the surface. In terms of the principal radii, the mean curvature is $H=\frac{1}{2}\left(\frac{1}{R_{1}}+\frac{1}{R_{2}}\right)$, and the Gaussian curvature is $K=\left(\frac{1}{R_{1}}\right)\left(\frac{1}{R_{2}}\right)$. A general form of $f_{c}$ to this order is given by [18: $f_{c}=2 \kappa\left(H-c_{0}\right)^{2}+\bar{\kappa} K$, where $\kappa$ and $\bar{\kappa}$ are constants usually denoted by 'bending rigidities'. The spontaneous curvature of the surface, $c_{0}$, is taken as zero for the rest of this discussion, because the film is assumed symmetric with respect to its mid-plane.

To find out the effects of polymer confinement on $\kappa$, and $\bar{\kappa}$, we first consider a vesicle (a spherical shell in $(D=3)$ ), with a small curvature, i.e. a shell of radius $a$, with a finite thickness $d$, where $d \ll a$. For a sphere both principal radii are equal to $a$, and we find $f_{c}=\frac{1}{2} \kappa\left(\frac{2}{a}\right)^{2}+\bar{\kappa}\left(\frac{1}{a}\right)^{2}$. Next consider a cylindrical shell $(D=2)$; now $R_{1}=a$, but $R_{2}=\infty$. Thus, $f_{c}=\frac{1}{2} \frac{\kappa}{a^{2}}$. Defining $\Phi_{M}=M / V=M /(S d)+\mathrm{O}(\epsilon), S$ being the surface area, and using the results for $\Delta F$ for the sphere and the cylinder we conclude that: 


$$
\begin{gathered}
\delta \kappa=\left(k_{B} T\right)\left(\Phi_{M} d^{3}\right)\left(\frac{1}{8}\left(1-f(k)-\frac{8}{\pi^{2}} h(k)\right)-\frac{k}{2 \pi^{2}}\right), \\
\delta \bar{\kappa}=\left(k_{B} T\right)\left(\Phi_{M} d^{3}\right)\left(-\frac{1}{6}+\frac{2}{\pi^{2}} h(k)\right),
\end{gathered}
$$

where $\delta \kappa$ and $\delta \bar{\kappa}$ are the changes in the bending rigidities. These expressions can be expanded in G.S.D. and C.L., to give:

$$
\begin{gathered}
\delta \kappa=-\left(k_{B} T\right)\left(\Phi_{M} l^{3}\right) \begin{cases}N \frac{1}{12}\left(\frac{d}{l}\right) & ; k>1 \\
N^{\frac{3}{2}} \frac{1}{9 \sqrt{6 \pi}}+\cdots & ; k<1\end{cases} \\
\delta \bar{\kappa}=+\left(k_{B} T\right)\left(\Phi_{M} l^{3}\right)\left\{\begin{array}{l}
N \frac{1}{6}\left(\frac{d}{l}\right) \\
\sqrt{N} \frac{1}{3} \sqrt{\frac{2}{3 \pi}}\left(\frac{d}{l}\right)^{2}-N \frac{3 \pi-8}{18 \pi}\left(\frac{d}{l}\right)-N^{\frac{3}{2}} \frac{1}{9}\left((3 \pi-8) \sqrt{\frac{2}{3 \pi^{3}}}-\sqrt{\frac{2}{3 \pi}}\right)+\cdots ; k<1
\end{array}\right.
\end{gathered}
$$

We have not considered any interactions between monomers. We expect that interactions between chains in a dense solution would screen out the effect of the walls on the concentration profile over a very short distance and hence weaken this curvature effect. We are currently studying this situation. But our results may hold qualitatively for concentrations below the overlap concentration, $\Phi_{m}^{*}$. To be consistent with our analysis we use Gaussian exponents to estimate $\Phi_{m}^{*}$ : In G.S.D. we require $N \Phi_{m}^{*}=\frac{N}{R_{F}^{2} d}=\frac{1}{l^{2} d}$. Thus $\Phi_{m}^{*}=\frac{1}{N l^{2} d}$. In C.L. the walks are three dimensional and we require $N \Phi_{m}^{*}=\frac{N}{R_{F}^{3}}=\frac{1}{\sqrt{N l^{3}}}$. Thus $\Phi_{m}^{*}=\frac{1}{N^{(3 / 2) l^{3}}}$. This leads to:

$$
\begin{gathered}
\frac{\delta \kappa}{\left(k_{B} T\right)}=- \begin{cases}\frac{1}{12} & ; k>1 \\
\sim 0.026 & ; k<1\end{cases} \\
\frac{\delta \bar{\kappa}}{\left(k_{B} T\right)}=+ \begin{cases}\frac{1}{6} & ; k>1 \\
0.06 \frac{1}{N}\left(\frac{d}{l}\right)^{2} & ; k<1\end{cases}
\end{gathered}
$$

Edwards theory assumes slow variations of the propagator [16], and is therefore valid over large length scales compared to the lattice spacing, $l$, and moderately large number of 
steps, $N$. (Aside: We also need $d \ll a$, in order to use our perturbative result). Notice that for $k<1$ we have $\frac{1}{N}\left(\frac{d}{l}\right)^{2}>1$ - which can be achieved e.g. with $N \sim 100$, and $d / l \sim 20$, then this term gives a factor of 4 . Estimating the bare rigidities of the box to be of the order of $k_{B} T$, we see that the effect is about $1 \%$ for $\kappa$, and $5 \%$ for $\bar{\kappa}$.

We emphasize that the major result of this article is that polymer confinement might reduce $\kappa$, and thereby induce spontaneous curvature in the system, such as transitions from lamellar to bicontinuous phases or tubules etc, provided that the effect persists after the inclusion of self-avoidance, i.e. monomer-monomer interactions, in the analysis. We expect that this would be the case below the overlap threshold, but future work should address this issue in detail. We note here that, since there are two length scales in the problem, one cannot solve the self-avoiding walk problem by simple scaling arguments.

After one understands the role of interactions, one should also consider the case of a polymer with a finite persistence length. We expect that in the regime where the radius of curvature is much larger than the persistence length the result would approach the one for the totally flexible polymer case we treated in the present article (modulo interactions). But when the two lengths are comparable, the aversity to bending of rigid polymers should modify the effect computed here.

Note added in proof: As we were preparing to publish we received a preprint by E. Eisenriegler, A. Hanke, and S. Dietrich, in which the authors also compute bending rigidities of systems with chains near a repulsive surface.

\section{ACKNOWLEDGMENTS}

We would like to thank C. Marques for useful discussions on several issues. KY and PP were supported by the National Science Foundation under UCSB MRL, DMR-9123048, and the Department of Energy under DOE DE-FGO3-87ER45288. TW and FS acknowledge support from the National Science Foundation under contracts DMR 92-08527 and DMR DMR-9400379. 


\section{APPENDIX A: DETAILS OF THE FREE ENERGY COMPUTATION:}

We employ Eq. (6) in three dimensions, which involves integrals that are trivial to do, and we find for $B_{n}$ the following expression:

$$
B_{n}(a, d)=\frac{8}{n^{2} \pi^{2}}\left\{\begin{array}{l}
1+g(\epsilon) ; n=\text { odd } \\
-3 g(\epsilon) ; n=\text { even }
\end{array}\right.
$$

where $g(\epsilon)=-\frac{1}{4} \frac{\epsilon^{3}}{(1+\epsilon)^{3}-1}$. Using this in Eq. (幽 results in Eq. (10), where $f$ is defined as follows:

$$
f(k) \equiv \frac{\sum^{(e)} \frac{e^{-k n^{2}}}{n^{2}}}{\sum^{(o)} \frac{e^{-k n^{2}}}{n^{2}}}
$$

where the symbol $\sum^{(e)}$ denotes a sum over all positive even $n,(n=2,4,6, \ldots)$; and $\sum^{(o)}$ is over all positive odd $n,(n=1,3,5, \ldots)$. As emphasized in the text Eq. (10) is exact with the function $f$ defined as above. This function can be expanded for large arguments (g.s.d. regime) as:

$$
f(k)=\frac{1}{4} e^{-3 k}\left(1+\mathrm{O}\left(\mathrm{e}^{-8 \mathrm{k}}\right)\right)
$$

For small arguments one can also expand $f$ with a little more work. First we define the function $A(t) \equiv \sum_{n=-\infty}^{\infty} e^{-t n^{2}}$. This function has the following property:

$$
A(t)=\sqrt{\frac{\pi}{t}} A\left(\frac{\pi^{2}}{t}\right)
$$

Now we define $E(k) \equiv \sum^{(e)} \frac{e^{-k n^{2}}}{n^{2}}$, and $O(k) \equiv \sum^{(o)} \frac{e^{-k n^{2}}}{n^{2}}$. Then $f(k)=\frac{E(k)}{O(k)}$. (Aside: $\frac{8}{\pi^{2}} O(k)$ is the partition function for the flat strip). One can easily verify that $E(0)=\frac{\pi^{2}}{24}$, and $O(0)=\frac{\pi^{2}}{8}$. Also, $\frac{\partial E(k)}{\partial k}=-\frac{1}{2}(A(4 k)-1)$, and $\frac{\partial O(k)}{\partial k}=-\frac{1}{2}(A(k)-A(4 k))$. Using Eq.( $\mathrm{A} 4)$, and the fact that $A(t)=1+\mathrm{O}\left(\mathrm{e}^{-\mathrm{t}}\right)$, when $k$ is large, one finds that $\int_{0}^{k} d t A(t)=$ $2 \int_{0}^{k} d t A(4 t)=2 \sqrt{\pi k}+\mathrm{O}\left(\mathrm{e}^{-\frac{\pi^{2}}{\mathrm{k}}}\right)$, when $k$ is small. This allows an expansion of $f$ :

$$
f(k)=\frac{1}{3} \frac{1-12 \pi^{-2}(\sqrt{\pi k}-k)}{1-4 \pi^{-2} \sqrt{\pi k}}+\mathrm{O}\left(\mathrm{e}^{-\frac{1}{\mathrm{k}}}\right) .
$$

This function crosses over to $\frac{1}{4} e^{-3 k}$ at $k \sim 0.3$. 
Notice that these expressions satisfy the consistency checks: $\epsilon=0 \Rightarrow B_{n}(d)=\frac{8}{n^{2} \pi^{2}}$, if $n$ is odd, and $B_{n}=0$, if $n$ is even. Also, $k=0 \Rightarrow \Delta F=0$.

In two dimensions the integrals are also exactly doable but the final expression for $B_{n}$ is now much more complicated - we do not bother including this here. It can, however, be expanded in $\epsilon$, to yield:

$$
B_{n}(a, d)=\frac{8}{n^{2} \pi^{2}} \begin{cases}1-\frac{1}{16} \epsilon^{2}+\frac{1}{2 \pi^{2} n^{2}} \epsilon^{2}+\cdots ; & n=\text { odd } \\ \frac{1}{16} \epsilon^{2}+\cdots & ; n=\text { even }\end{cases}
$$

Now, when one is not in G.S.D., one in principle needs to worry about the terms that depend on $n$ in the expansion of the energy spectrum, Eq.(9). But when the argument of the terms in question is larger than one, we are deep into the G.S.D. regime, whereas when it is smaller than one these terms can be expanded in $\epsilon$, giving only $\epsilon^{4}$ corrections. Thus, they can in practice be ignored when computing bending rigidities. Defining:

$$
h(k) \equiv \frac{\sum^{(o)} \frac{e^{-k n^{2}}}{n^{4}}}{\sum^{(o)} \frac{e^{-k n^{2}}}{n^{2}}}
$$

we find for the partition function the result in Eq. (11). Noticing that $-\frac{\partial h(k)}{\partial k}=O(k)$, and $h(0)=\frac{\pi^{2}}{12}$, one can expand $h$ using the expressions derived above:

$$
h(k)=\frac{\pi^{2}}{12} \frac{1-12 \pi^{-2} k+32 \pi^{-\frac{7}{2}} k^{\frac{3}{2}}}{1-4 \pi^{-\frac{3}{2}} \sqrt{k}}+\mathrm{O}\left(\mathrm{e}^{-\frac{1}{\mathrm{k}}}\right) .
$$

Again, these expressions satisfy the above-mentioned consistency checks. These expansions for $f$ and $h$ are in excellent agreement with numerical results for $0<k<\frac{1}{\pi}$. The results for the bending rigidities follow from these expansions. 


\section{REFERENCES}

[1] Duclos, P.; Exner, P. Rev. Math. Phys. 1995, 7, 73.

[2] Renger, W.; Bulla, W. Lett. Math. Phys. 1995, 35,1.

[3] Takagi, S.; Tanzawa, T. Prog. Theor. Phys. 1992, 87, 561.

[4] Clark, I.J.; Bracken, A.J. J. Phys. A 1996, 29, 339.

[5] Homma, T.; Innamoto, T.; Miyazaki, T. Phys. Rev. D 1990, 42, 2049.

[6] Jensen H.; Koppe, H. Ann. of Phys. 1971, 63, 586.

[7] daCosta, R.C.T. Phys. Rev. A 1981, 23, 1982.

[8] Ogawa, N.; Fujii, K.; Kobushukin A. Prog. Theor. Phys. 1990, 83, 894.

[9] Ogawa, N.; Fujii, K.; Chepilko, N.; Kobushukin, A. Prog. Theor. Phys. 1991, 85, 1189.

[10] Tolar, J. On a quantum mechanical d'Alembertian principle, in Group Theoretical Methods in Physics, Lecture Notes in Phys. 313, (Springer Verlag, Berlin, 1988), pp. $268-274$.

[11] Marcus, J. J. Chem. Phys. 1966, 45, 4493.

[12] Exner, P.; Seba, P.; Stovicek, P. Phys. Lett. A 1990, 150, 179.

[13] Goldstone, J.; Jaffe, R.L. Phys. Rev. B, 1992, 45, 14100.

[14] Switkes, E.; Russell, E.L.; Skinner, J.L. J. Chem. Phys. 1977, 67, 3061.

[15] Edwards S.F.; Doi, M. The Theory of Polymer Dynamics (Oxford Science Publications, New York, NY, 1986), p.19.

[16] deGennes, P.G. Scaling Concepts in Polymer Physics (Cornell University Press, Ithaca, NY, 1979).

[17] Yaman, K.; Marques, C. Unpublished results. 
[18] Safran, S.A. Statistical Thermodynamics of Surfaces, Interfaces, and Membranes (Addison-Wesley Publishing Co., 1994), p.189. 


\section{FIGURE CAPTIONS}

Fig.1 : Twisting tube example: solid cylinder $\longleftrightarrow$ torus.

Fig.2: $D=2$ annulus.

Fig. $3: D=3$ annulus.

Fig.4 : Numerical solution for $\tilde{E}(1 / \epsilon)$, in two, four, and five dimensions. 


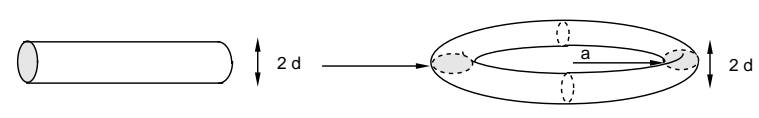




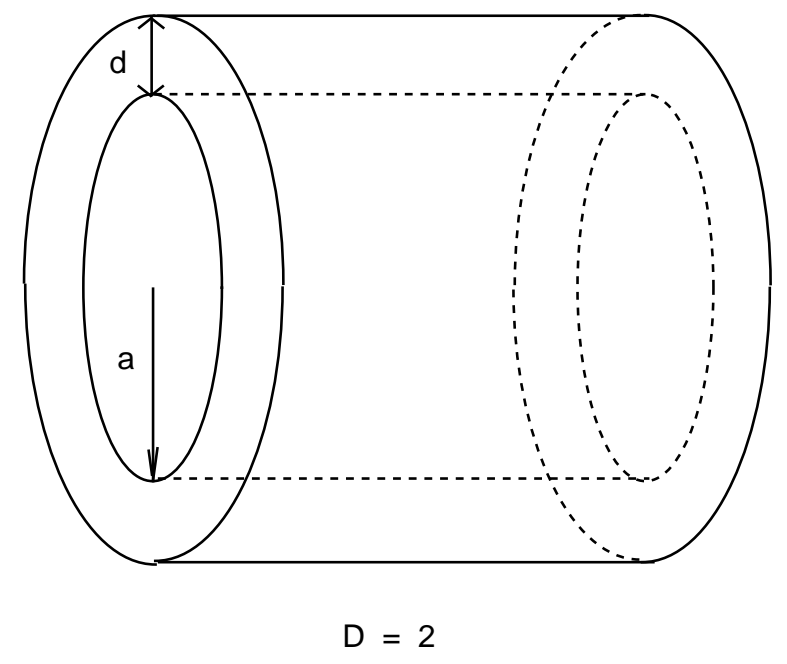




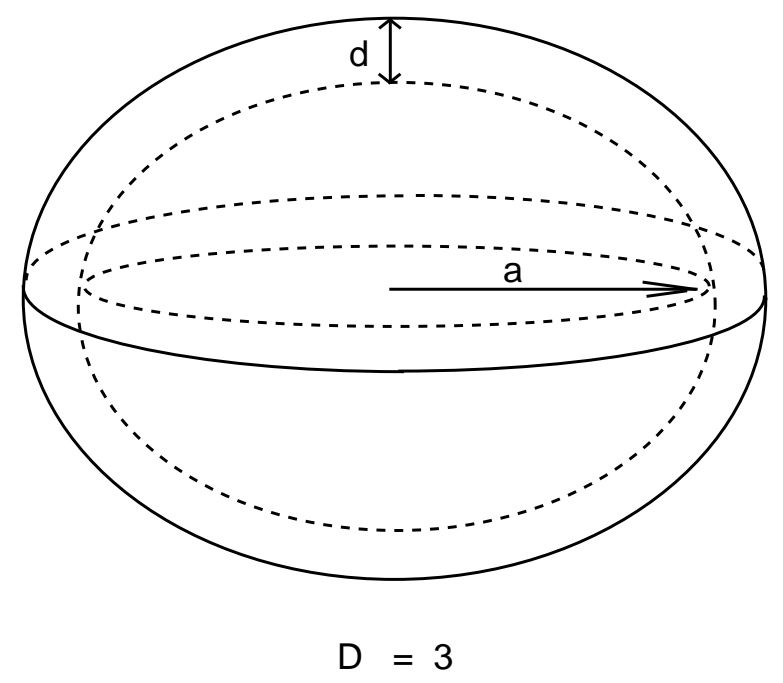




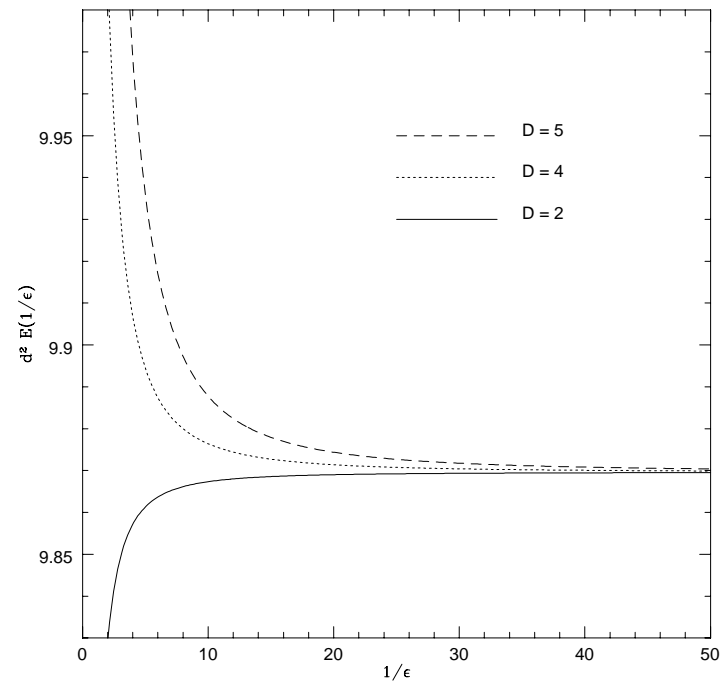

